\title{
Interpretation of Electron Rutherford Backscattering Spectrometry for Hydrogen Quantification
}

\author{
Rafael Alvarez, Francisco Yubero*
}

Instituto de Ciencia de Materiales de Sevilla (CSIC - Univ. Sevilla) Av. Américo

Vespucio 49, E-41092 Sevilla, Spain (http://sincaf-icmse.es)

*Corresponding author: yubero@icmse.csic.es

\begin{abstract}
In the last few years several papers have appeared showing the capabilities of Electron Rutherford Backscattering Spectrometry (eRBS) to quantify the $H$ content at surfaces. The basis of the $\mathrm{H}$ detection in this technique relies on the difference in recoil energy of the incident electrons depending on the mass of the atoms located at the surface that act as scatter centers. In this paper we address the interpretation of eRBS spectra of Hydrogen containing surfaces. The aim is to compare the naïve single elastic scattering approximation with a more realistic description of eRBS spectra including multiple elastic scattering using the HQeRBS (Hydrogen Quantification eRBS) software based on a Monte Carlo algorithm. It is concluded that multiple elastic scattering is a significant contribution to experimentally measured eRBS spectra of a polyethylene surface. It induces significant broadening of the distribution of the maximum elastic scattering angle along the electron trajectories contributing to the measured spectra. However, it has weak effect in the energy distribution of the collected electrons (about $10 \%$ overestimation of the $\mathrm{H}$ content in the particular case of a polyethylene surface with respect to the corresponding ratio of elastic scattering cross sections).
\end{abstract}




\section{Introduction}

The quantitative determination of the Hydrogen $(\mathrm{H})$ content at surfaces is a subject of key importance in many technological fields. Thus, surface functionalization, polymers, carbon based hard coatings or new $\mathrm{H}$ storage materials, may require such analysis to improve the understanding of the processes that involve the presence of $\mathrm{H}$ atoms at surfaces.

However, quantification of this element at the surface region (few nanometres depth) of a sample is not an easy task. Note for example that direct evidence of $\mathrm{H}$ atoms does not show up in standard non destructive surface analysis techniques such as X-ray photoemission or Auger spectroscopies. Indirect way to quantify $\mathrm{H}$ content at the surface region can be made by means of High resolution electron energy loss spectroscopy (HREELS). However, in this case, only those $\mathrm{H}$ atoms that contribute to the vibrational absorption spectra are observed. Secondary ion mass spectroscopy (SIMS) is another indirect way to determine $\mathrm{H}$ content through the analysis of the radicals present at surfaces, but this technique has as the drawbacks that it is destructive and that its interpretation is handicapped by strong matrix effects. It is also possible $\mathrm{H}$ quantification with surface sensitivity by means of $1 \mathrm{H}(15 \mathrm{~N}, \alpha) 12 \mathrm{C}$ nuclear reaction analysis, but this technique is not easily available.

Electron Rutherford backscattering spectrometry (eRBS) using primary electron beams of 1-2 keV has been postulated as an alternative technique to quantify the $\mathrm{H}$ content at the surface of a-C:H and polymer samples [1-3]. This is due to the fact that the energy distribution of elastically backscattered electrons at surfaces can be correlated with their $\mathrm{H}$ content. The recoil energy of the impinging electrons depends on the atomic mass of the particular atom present at the surface, and the difference in recoil energies between the $\mathrm{H}$ atoms and the rest of the elements present at the surface of the analysed sample is easily observed with any standard electron spectrometer used in surface analysis.

Despite of the capabilities of this technique to quantify $\mathrm{H}$ content at surfaces, its use is not extensive. Only few groups have reported scientific papers using it [18]. Following the same principles but using higher electron kinetic energies (up to 
$40 \mathrm{keV}$ ), this technique has also been proposed to evaluate electron inelastic mean free paths and to study of binary compounds and buried interfaces [9-12].

This paper aims to give a deep inside look into the interpretation of eRBS spectra, focussing on the understanding of multiple elastic scattering angle effects on the electron trajectories contributing to eRBS spectra of $\mathrm{H}$ containing surfaces. This is done by means of HQ_eRBS (Hydrogen Quantification by eRBS) Monte Carlo software, specially developed for this purpose. The developed software will be freely available for the scientific community (for non-commercial use) to encourage the practical use of this technique.

It is worth mentioning that experiments with elastically backscattered electrons are extensively used to determine electron inelastic mean free paths [13]. In this context the experimental technique is known as elastic peak electron spectroscopy (EPES). However, we prefer to keep the eRBS acronym here due to the similarity of the technique with traditional Rutherford Backscattering spectrometry experiments with $\mathrm{MeV}$ ions.

\section{Brief description of the theoretical background}

A first naïve description of the electron-solid interaction can be made considering binary electrostatic collisions between the impinging electrons and the nuclei of the atoms in the sample. Within a classical description of the interaction of charged particles, considering energy and momentum conservation laws, the energy transferred (recoil energy $E_{r n}$ ) of an electron impinging on a surface of a multi-elemental material $\left(M_{n}\right.$ : atomic mass of the sample atoms) can be written as [14]:

$$
E_{r n}=\frac{2 m}{M_{n}} E_{0}\left[1-\cos \theta+\sqrt{\frac{M_{n} \varepsilon_{n}}{m E_{0}}}(\cos \alpha-\cos \alpha \cos \theta-\sin \theta \sin \alpha \cos \varphi)\right]
$$

where $\theta$ is the angle of scattering, $m$ and $E_{0}$ are the mass and kinetic energy of the electron before scattering, $\varepsilon_{n}$ is the kinetic energy of the atom, and $\alpha$ and $\varphi$ characterizes the direction of motion of the atom with respect to the velocity of 
the electron before the scattering event and the scattering plane. If the scatter atom is at rest (i.e., $\varepsilon=0$ ), the well-known energy transfer relation of an atom at rest is recovered

$$
E_{r}=\frac{2.18 \times 10^{-3}}{M} \sin ^{2}(\theta / 2) E_{0}
$$

( $E_{0}$ given in $\mathrm{eV}$ ). Within this approach, differences in electron recoil energies $\Delta E_{\mathrm{r}}$ between two different atoms at the surface of a given sample will be given by:

$$
\Delta E_{r}=2.18 \times 10^{-3} \sin ^{2}\left(\frac{\theta}{2}\right) E_{0}\left[\frac{1}{M_{1}}-\frac{1}{M_{2}}\right]
$$

According to eqn.(3), we will observe recoil energy differences in the order of 2$4 \mathrm{eV}$ for multi-elemental surfaces composed by $\mathrm{H}\left(M_{1}=1\right)$ and other elements (for example $C$ with $M_{2}=8$ ), for scattering angles of more than $120^{\circ}$ and kinetic energies in the 1-2 keV energy range. Thus, the observation of several elastic peaks in a eRBS experiment is an evidence of the presence of different type of atoms at the sample surface.

On the other hand, to perform elemental quantification at the surface region the corresponding electron scattering cross section will need to be considered. In this case we have used elastic scattering cross section obtained from NIST Electron Elastic-Scattering Cross-Section Database [15]. If only independent single scattering events are considered, the stoichiometry of binary homogeneous samples will be directly correlated to the intensity ratio of the corresponding electron backscattering signals. The ratio of cross sections $\sigma \mathrm{c} / \sigma_{\mathrm{H}}$ for electrons of $1500 \mathrm{eV}$ impinging on $\mathrm{C}$ and $\mathrm{H}$ takes values about 42 for scattering angles of $135^{\circ}$.

\section{Multiple elastic scattering approximation: Monte Carlo simulations using HQ_eRBS code}

Monte Carlo (MC) simulation of electron transport in solids is based on the stochastic description of scattering processes. Binary collisions are considered according to the description outlined above. Electron penetration is approximated 
by a classical zigzag trajectory. Besides, angular differential elastic scattering cross sections are used to account for the probability of scattering as a function of the energy of the electron and scattering angle. They are described by the partial expansion method using Hartee-Fock-Slater type wavefunctions [15].

We suppose that the studied sample is semi-infinite, homogenous and amorphous. The scattering point is where the electron changes its direction and/or energy. The primary energy beam is considered monochromatic. In our calculations both the elastic and inelastic scattering events are taken into account. If an electron suffers an inelastic collision, the calculation of its trajectory is stopped, since it will not contribute to the elastic peak spectrum. Particular values of scattering angles of electrons in an individual event are realized by random numbers following the angular differential elastic cross sections of the target material.

After each elastic scattering event, the recoil energy is calculated according to eqn.(1), which takes into account the mass of the scattering atom. Thus energy and angular distributions of elastically backscattered electrons from a semiinfinite sample were determined. This was done using the HQ_eRBS Monte Carlo code developed to simulate eRBS spectra of $\mathrm{H}$ containing surfaces.

In this work we present results on polyethylene $\left(\mathrm{CH}_{2}\right)$. This material is chosen because the $\mathrm{H}$ content is higher than in most other polymers. We select the primary electron energy as $E_{0}=1.5 \mathrm{keV}$ with an incident angle of $\theta_{\text {in }}=45^{\circ}$ with respect to the sample's surface normal and detection normal to the surface. Analyzer acceptance angle was assumed to be $\theta_{a c}= \pm 9$. The energy spread of the primary electron beam is simulated by Gaussian distribution with a full width at half maximum of $0.5 \mathrm{eV}$. The other input data of our Monte Carlo simulations were as follow: The atomic density is $\rho\left(\mathrm{CH}_{2}\right)=0.93 \mathrm{~g} / \mathrm{cm}^{3}$. The corresponding inelastic mean free paths is $\lambda_{\text {in }}\left(\mathrm{CH}_{2}\right)=53 \AA$ [16]. We used $\bar{\varepsilon}=120 \mathrm{meV}$ and $\bar{\varepsilon}=$ $80 \mathrm{meV}$ for the average kinetic energy of the $\mathrm{H}$ and $\mathrm{C}$ atoms, respectively [4]. The number of incident primary electrons was in the range of $10^{10}$.

\section{Results and discussion}


In the following we address several points concerning the interpretation of eRBS spectra. The aim is to compare the naïve single scattering approximation outlined above with a more realistic description of simulated eRBS spectra including multiple elastic scattering.

A first aim of this study is to give a clear image of the trajectories of the backscattered electrons contributing to measured eRBS spectra.

\section{Multiple scattering contributions to eRBS spectra}

Figure 1 shows $\mathrm{HQ}_{\text {_eRBS }} \mathrm{MC}$ simulations of electrons backscattered from a $\mathrm{CH}_{2}$ sample ( $E_{0}=1500 \mathrm{eV}$ kinetic energy, $45^{\circ}$ angle of incidence, normal detection, 9 은 acceptance angle). The figure discriminates between the contributions to the total backscattered spectra of single and multiple scattering collisions. The " $\mathrm{C}$ " and " $\mathrm{H}$ " peaks can easily be identified appearing at about 0.3 and $2.7 \mathrm{eV}$ recoil energies, respectively. About $40 \%$ of the intensity of the total spectra is formed by electrons whose trajectory has experienced just a single elastic scattering event, either at $\mathrm{C}$ or $\mathrm{H}$ atoms. Electron trajectories composed by several elastic scattering collisions on $\mathrm{H}$ atoms only account for less than $5 \%$ of the " $\mathrm{H}$ " peak. However multiple $\mathrm{C}$ collisions contribute to about $50 \%$ of the intensity of the " $\mathrm{C}$ " peak. On the other hand, the contribution of mixed multiple collisions accounts for less than $7 \%$ at the " $\mathrm{C}$ " peak and about half of the " $\mathrm{H}$ " peak. Note also that there is significant intensity between the $\mathrm{C}$ and $\mathrm{H}$ peaks in the mixed $\mathrm{C} / \mathrm{H}$ contribution

An important point for quantification purposes is the ratio between peaks ascribed to presence of $\mathrm{H}$ and $\mathrm{C}$ at the sample surface. In the particular case described in figure 1 , least squares fitting of the $\mathrm{H}$ and $\mathrm{C}$ peaks of the total spectrum by Gaussian functions gives a $\mathrm{C} / \mathrm{H}$ intensity ratio of 19 , while the predicted ratio from the elastic scattering cross section is 21 . This $10 \%$ deviation is due to the contribution of mixed multiple scattering to the intensity between the peaks, which provokes overestimation of the $\mathrm{H}$ signal. This result will have to be taken into account for accurate $\mathrm{H}$ quantification from eRBS measurements.

Figure 2 shows a 2D plot of $\mathrm{HQ}$ eRBS MC simulation where the number of elastic collisions vs the recoil energy is shown $\left(\mathrm{CH} 2,1.5 \mathrm{keV}\right.$, normal detection, $45^{\circ}$ 
incident angle, 9o acceptance angle). The color scale (log scale) indicates the number of electrons detected for each recoil energy-number of elastic collisions pair. It is worth noting that the $C$ peak includes electrons which underwent more multiple collisions (up to 10-12) than the $\mathrm{H}$ peak (up to 4-5).

Figures 1 and 2 show that the electron trajectories contributing to experimental eRBS spectra are strongly affected by multiple elastic scattering. However, these multiple elastic scattering effects introduce only a $10 \%$ deviation on the $\mathrm{C} / \mathrm{H}$ intensity ratio, allowing the use of the $\mathrm{H}$ and $\mathrm{C}$ intensities as a first reasonable estimation for elemental quantification purposes.

\section{Angular dispersion of the backreflected electron trajectories}

Figure 3 shows the distributions of $\theta_{\max }$, the highest scattering angle among all scattering angles during the trajectory of an electron which is finally detected. The results are sorted according to the type of elastic collisions contributing to the total eRBS spectrum.

Single- $\mathrm{C}$ and single- $\mathrm{H}$ distribution of $\theta_{\max }$ are forced by the analyzer acceptance angle. In these cases, the electrons reaching the detector follow strict V-type trajectories and no events are allowed out of the limits $180-\theta_{i}-\theta_{a c}<\theta_{\max }<180$ $-\theta_{i}+\theta_{a c}$. This is not the case for electron trajectories characterized by several elastic scattering events. In fact long tails are observed in the $\theta_{\max }$ distributions out of the $135^{\circ} \pm \theta_{\text {ac }}$ limits. However, still most of the intensity is within $135^{\circ} \pm 20^{\circ}$ limits, so most trajectories contributing to the eRBS spectra can still be considered V-type. Similar conclusion was deduced for those electrons contributing to the single inelastic scattering cross section in reflection electron energy loss spectra in a previous work [17].

Figure 4 shows HQ_eRBS MC simulations of $\theta_{\max }$ vs recoil energy detected for each type of electron trajectory. The color scale (log scale) indicates the number of electrons detected for each recoil energy/maximum scattering angle pair. The figure shows single collisions at $\mathrm{H}$ (a) or $\mathrm{C}$ (c) atoms, multiple collisions at either $\mathrm{H}$ (b) or $\mathrm{C}$ atoms (d), as well as the mixed $\mathrm{H} / \mathrm{C}$ multiple collisions contribution (e) to the total spectrum $(\mathrm{f})$. This series of $2 \mathrm{D}$ drawings gives us a clear picture of the electron trajectories contributing to eRBS spectra from $\mathrm{CH}_{2}$. Note that multiple 
scattering just at $\mathrm{C}$ atoms is a significant contribution to the spectrum and the corresponding electron trajectories may differ significantly from each other (note the large dispersion in the $\theta_{\max }$ values). The most probable $\theta_{\max }$ is forced by the experiment geometry (incidence, detection, and acceptance angles), but the dispersion of $\theta_{\max }$ increases significantly when multiple scattering is considered. Note also that electron trajectories of backscattered electrons on either $\mathrm{H}$ or $\mathrm{C}$ atoms may differ significantly. Thus, broader angular dispersion is expected for those electrons contributing to the " $\mathrm{C}$ " peak than to the " $\mathrm{H}$ " peak. It is also worth noting that most mixed $\mathrm{H} / \mathrm{C}$ elastic scattering contribution adds intensity to the "C" peak (see also Figure 1). Besides, note that there is some intensity inbetween of the $\mathrm{H}$ and $\mathrm{C}$ peak contribution that cannot be clearly assigned to $\mathrm{H}$ or $C$ (with maximum scattering angle significantly away from $135^{\circ}$ ). This intensity is responsible for the $10 \%$ deviation observed above between the quantification of simulated eRBS spectra by fitting with symmetric Gaussian peaks and the predicted intensity ratio according to the elastic scattering cross sections. Most probably better description of the $\mathrm{H}$ and $\mathrm{C}$ peaks would be as slightly asymmetric with broader tails in the energy region between the two main recoil energies corresponding to the $\mathrm{H}$ and $\mathrm{C}$ contributions, with more intensity in the $\mathrm{C}$ peak, due to the higher probability for elastic scattering.

\section{Conclusions}

Multiple elastic scattering is a significant contribution to experimentally measured eRBS spectra of a polyethylene surface. It mainly induces broadening of the distribution of the maximum scattering angle of elastic scattering of the electron trajectories contributing to the measured spectra with low effect (about 10\% overestimation of the $\mathrm{H}$ content in the particular case of a polyethylene surface with respect to the corresponding ratio of elastic scattering cross sections) in the energy distribution of the collected electrons. Thus, elemental $\mathrm{H}$ quantification based on eRBS measurements can be done by just making normalized intensity ratio between $\mathrm{H}$ and $\mathrm{C}$ contributions to the spectra. Finally, we would like to mention that the HQ_eRBS MC code used in this work, especially developed to improve the understanding of eRBS spectra of $\mathrm{H}$ containing surfaces, will be available free of charge to the scientific community for non-commercial use. 


\section{References}

[1] G.T. Orosz, G. Gergely, M. Menyhard, J.Toth, D. Varga, B. Lesiak, A. Jablonski, Surf. Sci. 2004; 566-568, 544.

[2] F. Yubero, V.J. Rico, J.P. Espinós, J. Cotrino, A.R. González-Elipe, Appl. Phys. Lett. 2005; 87, 084101.

[3] V.J. Rico, F. Yubero, J.P. Espinós, J. Cotrino, A.R. González-Elipe, D. Garg, S. Henry, Diamond and Relat. Mater. 2007; 16, 107.

[4] D. Varga, K. Tokesi, Z. Berenyi, J. Toth, L. Kover, Surf. Interface Anal. 2006; $38,544$.

[5] B. Lesiak, J. Zemek, J. Houdkova, Polymer 2008; 49, 4127.

[6] M. Filippi, L. Calliari, C. Verona, G. Verona-Rinati, Surf. Sci. 2009; 603, 2082.

[7] J. Zemek, J. Houdkova, P. Jiricek, H. Kozak, A. Kromka, Diamond Relat Mater. 2012; 26, 66.

[8] F. Yubero, K. Tokési, Appl. Phys. Lett. 2009; 95, 084101.

[9] M. Vos, M.R. Went, J. Electron Spect. Relat. Phenom. 2007; 155, 35.

[10] M. R. Went, M. Vos Appl. Phys. Lett. 2007; 90, 072104.

[11] M. Vos, M.R. Went Phys. Rev. B 2006; 74, 205407.

[12] K. Tokesi, D. Varga, Surf. Sci. 2010; 604, 623.

[13] S. Tanuma, T. Shiratori, T. Kimura, K. Goto, S. Ichimura, C. J. Powell, Surf. Interface Anal. 2005; 37, 833.

[14] D. Varga, K. Tokesi, Z. Berenyi, J. Toth, L. Kover, G. Gergely, A. Sulyok, Surf. Interface Anal. 2001; 31, 1019. 
[15] A. Jablonski, F. Salvat, C. J. Powell, NIST Electron Elastic-Scattering Cross-Section Database - Version 3.2, National Institute of Standards and Technology, Gaithersburg, MD (2010).

[16] S. Tanuma, C. J. Powell, D. R. Penn, Surf. Interf. Anal. 1994; 21, 165.

[17] F. Yubero, N. Pauli, A. Dubus, S. Tougaard, Phys. Rev. B 2008; 77, 245405.

\section{Figure captions}

Figure 1. HQ_eRBS MC spectra of $1.5 \mathrm{keV}$ electrons impinging on $\mathrm{CH}_{2}$ (normal detection, $45^{\circ}$ incidence angle, $9^{\circ}$ acceptance angle). Single $\mathrm{H}$, single $\mathrm{C}$, multiple $\mathrm{C}$, multiple $\mathrm{H}$ and mixed $\mathrm{H} / \mathrm{C}$ contributions to the total spectrum are shown.

Figure 2. HQ_eRBS MC simulation of the number of elastic scattering events vs the recoil energy $\left(\mathrm{CH}_{2}, 1.5 \mathrm{keV}\right.$, normal detection, $45^{\circ}$ incident angle, $9^{\circ}$ acceptance angle).

Figure 3. HQ_eRBS MC simulations of the distribution of $\theta_{\max }$, the highest scattering angle among all scattering angles during the trajectory of an electron which is finally detected. The results are sorted according to the type of elastic collisions contribution to the total eRBS spectrum. $\left(\mathrm{CH}_{2}, 1.5 \mathrm{keV}\right.$ primary energy, normal detection, $45^{\circ}$ incidence angle, $9^{\circ}$ acceptance angle).

Figure 4. HQ_eRBS MC simulations of the different contributions to the spectra, shown as maximum scattering angle vs recoil energy of $\left(\mathrm{CH}_{2}, 1.5 \mathrm{keV}\right.$ primary energy, normal detection, $45^{\circ}$ incident angle, $9^{\circ}$ acceptance angle). The color scale (log scale) indicates the number of electrons detected for each recoil energy-maximum scattering angle pair 
Figure 1

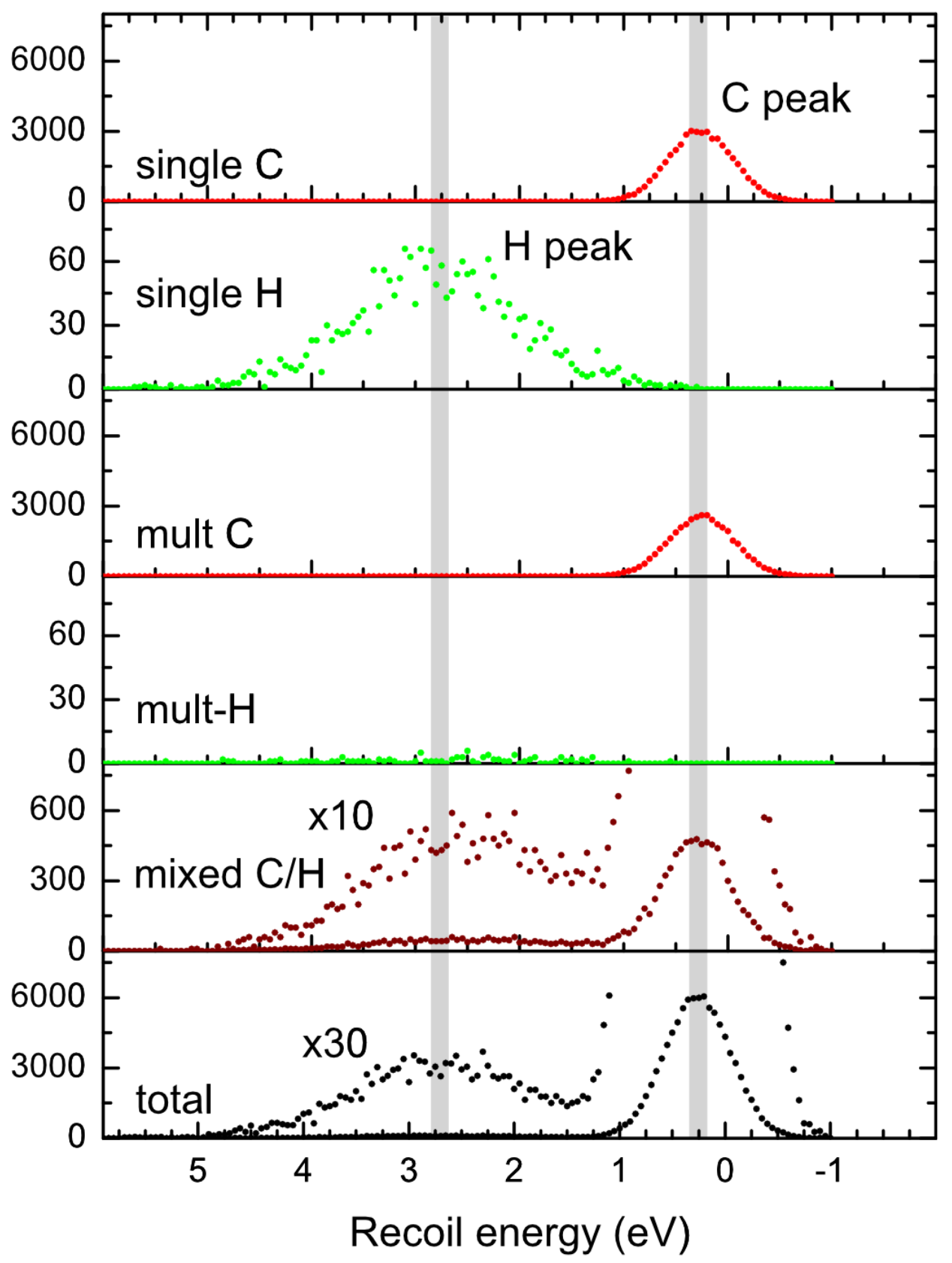


Figure 2

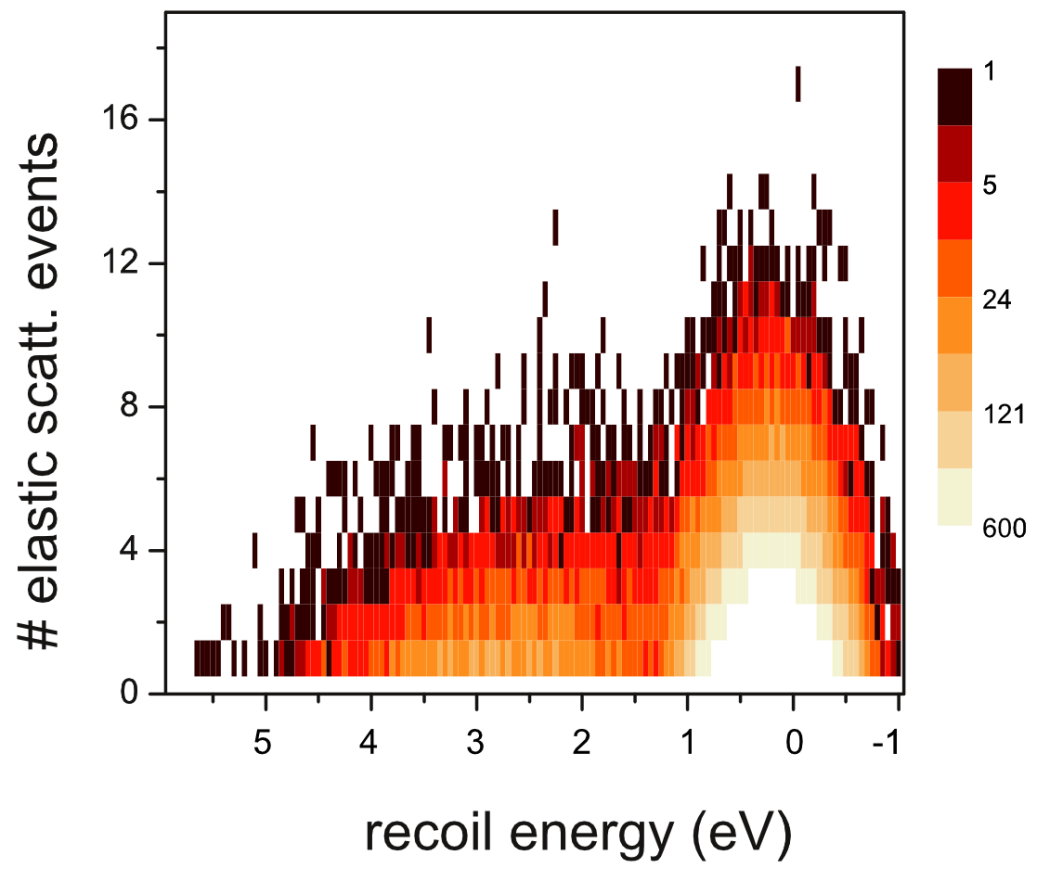


Figure 3

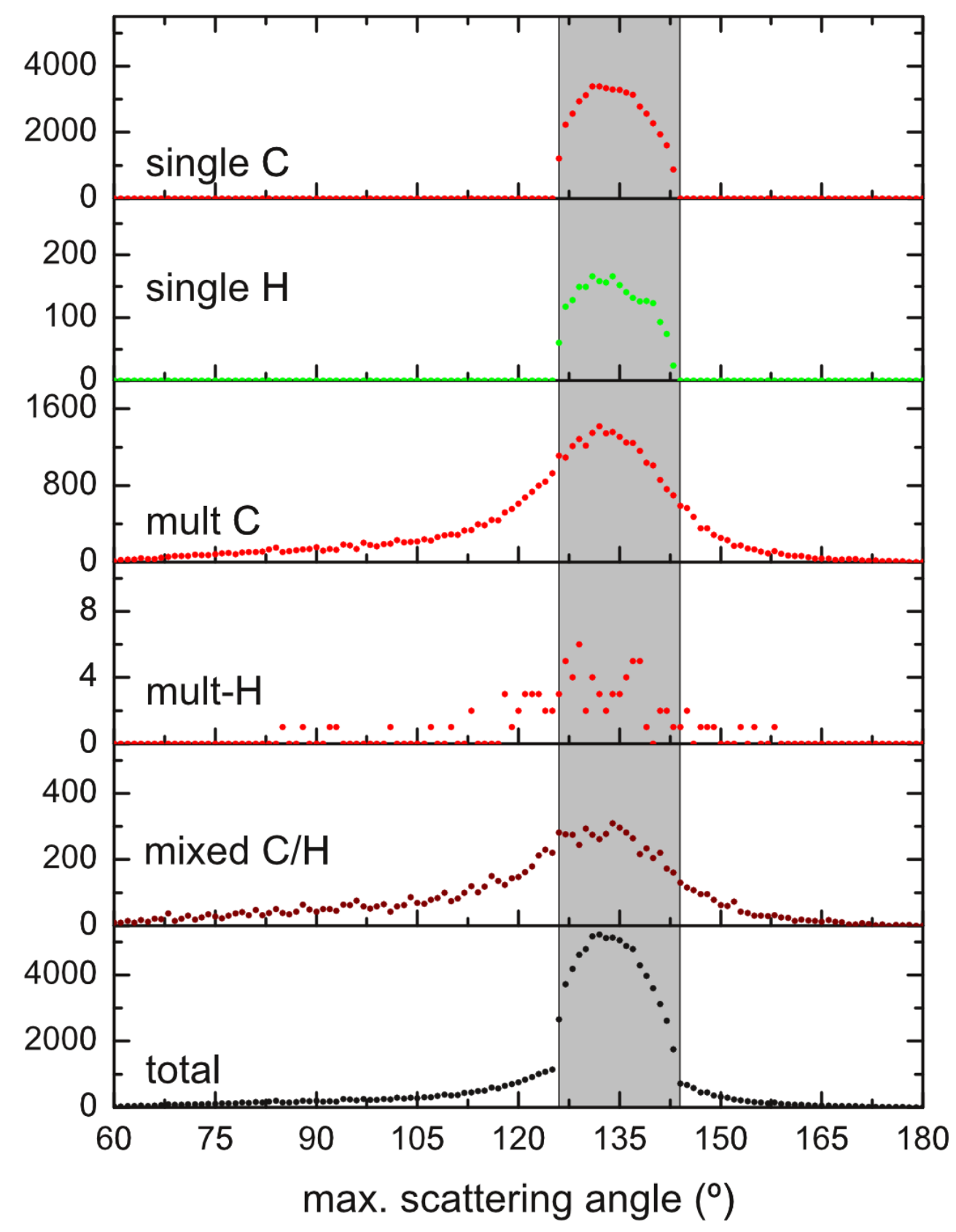


Figure 4
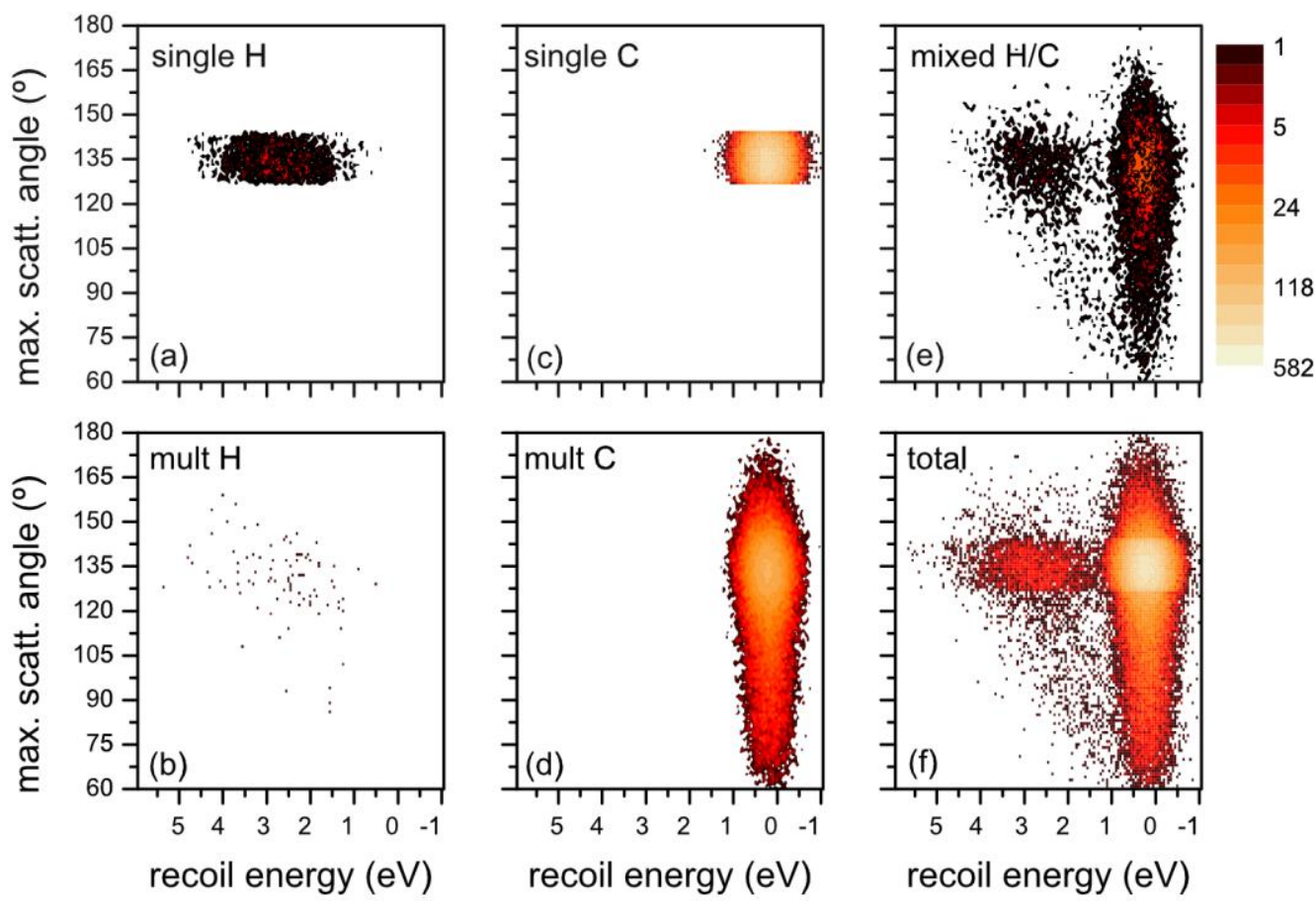